\title{
Technical Efficiency of Construction Companies: A Stochastic Frontier Model with Dependent Error
}

\author{
Roslah Arsad, Zaidi Isa, Ruzanna Ab Razak
}

\begin{abstract}
There are several problems with using the Standard Stochastic Frontier (SF) model to produce a company's technical efficiencies. One of the problem is the hypothesis of independence between the error components, known as, statistical noise and inefficiency term. Recent studies have used the SFA-copula model where the copula element captures the joint distribution of the two error components thus assuming that the errors are dependent. This research seeks to use the Cobb-Douglas Stochastic Frontier production model (standard model), SFA-copula models and DEA models (DEA-CCR and DEA-BCC) to compare the technical efficiencies of 12 publicly listed construction companies in Malaysia. The latter models are non-parametric frontier models which will be used as a base to determine the best SF model. The four copulas considered in this study are Clayton, Gumbel, A12 and Product copulas. The main findings of this study are the SFA-copula models showed consistency in terms efficiency scores and rankings of companies as compared to DEA models. The results further raise the question of the reliability of standard $S F$ model especially when SFA-product copula was chosen as the best model for measuring efficiency performance. Therefore, it is obvious that it is not possible to ignore the reliance between the noise and inefficiency term.
\end{abstract}

Keywords: Copula, company's performance, stochastic frontier, technical efficiency.

\section{INTRODUCTION}

Measuring the efficiency of decision-making units (DMUs) has a major role in economy and management. A technical efficiency (TE) for a firm or a DMU reflects the capability of that firm in achieving maximal output from a given set of inputs. A company is said to be technically effective if the highest output from the minimum input amount can be produced. The distance between the production function frontier and the observed production (DMU) is directly linked to inefficiency. This distance usually called inefficiency [1]. Besides that, TE can also affect a firm's competitive position. By comparing TEs of firms, one can determine which firm is most efficient in utilizing given inputs to produce maximum output. The popular tools used to achieve DMU effectiveness are Data

Revised Version Manuscript Received on September 16, 2019.

Roslah Arsad, Faculty of Computer and Mathematical Sciences, Universiti Teknologi MARA, Perak Branch, Tapah Campus, Tapah Road, 35400 Perak, Malaysia; School of Mathematical Sciences, Faculty of Sciences and Technology, Universiti Kebangsaan Malaysia, 43600 UKM Bangi, Selangor, Malaysia.

Zaidi Isa, School of Mathematical Sciences, Faculty of Sciences and Technology, Universiti Kebangsaan Malaysia, 43600 UKM Bangi, Selangor, Malaysia.

Ruzanna Ab Razak, Faculty of Management, Multimedia University, 63100 Cyberjaya, Selangor, Malaysia.
Envelopment Analysis (DEA) and Stochastic Frontier Analysis (SFA). DEA is a non-parametric frontier method and require minimal assumptions. They do not impose restrictions on the functional forms relating inputs and outputs. They assume that all deviation from the frontier is carried out by the inefficiency [2].

Conversely, the parametric SFA has distributional assumptions for the two types of error, noise, $v_{i}$ and technical inefficiency error, $u_{i}$. The error term specification is given by $\varepsilon=v_{i}-u_{i}$. In estimating the efficiency based on SFA models, researchers assume that error terms $u_{i}$ and $v_{i}$ are independent [3], [4]. Nevertheless, the validity of the independent errors assumption has been questioned by many researchers, particularly in the context of inefficiency situation. Distinguishing between sources of randomness that are controllable and those that are not in the SFA model argue by [5], however they keep independence assumption between technical inefficiency and the noise error component. There is no statistical or financial reason to orthogonally impose between $u_{i}$ and $v_{i}$, in reality, being consistently composed of independent components would be uncommon for a productive scheme.

For example, noisy, seasonal changes frequently disturb productive choices in agriculture. Another instance is the potential adverse effect of uncontrolled spillage of hazardous by product by the heavy industry polluter on its individual laboratory effectiveness. Study by [6] also claim that various crop production, natural calamity in one season may influence decision-making in the following season, and even management choices may be influenced by random error variables such as weather. This show that in some situations in efficiency at a time point may depend on the inefficiency or noise of past time points [7].

As a result, the method adopted here generalizes the statistical model supporting the SFA model by showing a framework of reliance that enables connection between $u_{i}$ and $v_{i}$. SFA's dependent error components are less restrictive than the standard SFA and therefore statistically more attractive. Thus a possible dependency between $u_{i}$ and $v_{i}$ suggested by [8]. The copula method was used to model the reliance and then it was possible to estimate parameters of the SFA model. It can lead to partial estimates of parameter and 
thus generates inaccurate technical efficiency by ignoring reliance between the error elements [9]. The study's primary goal is to compare the technical efficiencies of 12 companies generated by standard SFA model, SFA-copula model and DEA models. This research also offers an overview of overview of stochastic frontier analysis (SFA) and offers theoretical debate on the use SFA copulas and their benefits in estimating SFA parameters through modelling error terms. The remainder of the paper is organized as follows. Section II provides a brief description of stochastic frontier model, copula and its characteristics, and the stochastic frontier analysis based copula. Section III presents the methodology on the chosen dataset for estimating the models and Section IV provides the outcomes and debate. Finally, in the final chapter of paper, the conclusion and the future work are observed.

\section{LITERATURE REVIEW}

\section{A. Stochastic frontier analysis}

Stochastic frontier analysis (SFA) is a method of economic modelling, which was firstly introduced in 1977 [3], [4]. Standard parametric stochastic frontier models assume that there is a production function $f$ that converts $X \in \mathfrak{R}_{+}^{p}$, a vector of inputs of dimension $p$, into a scalar output $Y \in \mathfrak{R}_{+}$. If one has $n$ observations of $\left(X_{i}, Y_{i}\right)$, the model can be written for the $i^{\text {th }} \mathrm{DMU}$ as

$$
y_{i}=f\left(x_{i}, \beta\right)+\varepsilon_{i}, \quad i=1, \ldots \ldots n
$$

where $y_{i}=\log \left(Y_{i}\right), \quad x_{i}=\log \left(X_{i}\right), \beta$ is a vector of parameters to be estimated, and $\varepsilon_{i}$ is composite error term.

The function $y_{i}=f\left(x_{i}, \beta\right)$ is interpreted as the production frontier. The stochastic error term, $\varepsilon_{i}$ contains information about the noise and the inefficiency. It can decompose into technical inefficiency and a noise term, which can be estimated. A typical specification is given by:

$$
\varepsilon_{i}=v_{i}-u_{i}
$$

where $v_{i}$ is a normal error term, $v_{i} \sim N\left(0, \sigma_{v}^{2}\right)$ and $u_{i}$ is a inefficiency error term with non-negative value, $u_{i} \geq 0$. The different distribution types for $u_{i}$ have been proposed in literature are exponential, half-normal, truncated-normal and gamma distribution.

Random noise error component, $v_{i}$ affecting the production process not directly attributable to the producers or the underlying technology. The noise may come from weather changes, economic, adversities, etc.[10]. The other component, $u_{i}$ measure technical inefficiency in the sense that it measures the shortfall of output of $y_{i}$ from its maximal possible value given by the stochastic frontier, $y_{i}=f\left(x_{i}, \beta\right)+v_{i}$. One sided error term $u_{i} \geq 0$ allows the distinction between DMUs, for example, DMUs that are on the frontier have $u_{i}=0$ and others that are below the frontier have $u_{i}>0$. The stochastic model also permits to estimate $\beta$ and its standard errors. The parameters of the model described in (1) and (2) can be estimated using maximum likelihood method and $\varepsilon_{i}$ can be predicted by $\hat{\varepsilon_{i}}=y_{i}-f\left(x_{i}, \hat{\beta}\right)$, which contains information on $u_{i}$.

Since $T E_{i}$ denotes technical efficiency, therefore it can be writes as $T E_{i}=\exp \left(-u_{i}\right)$ where $u_{i} \geq 0$. In this study, function for $f\left(x_{i}, \beta\right)$ is log-linear Cobb-Douglas form, and can written as:

$$
\ln y_{i}=\beta_{0}+\sum \beta_{r} \ln x_{r i}+v_{i}-u_{i}
$$

where $v_{i} \sim N\left(0, \sigma_{v}^{2}\right)$ and $u_{i} \sim N^{+}\left(0, \sigma_{u}^{2}\right)$.

\section{B. Stochastic frontier models and copula}

Referring to (3), the SFA model assumes that $v_{i}$ and $u_{i}$ are dependent errors. In this study, we assume the normal distribution for $v_{i}$ case, $v_{i} \sim N\left(0, \sigma_{v}^{2}\right)$ and $u_{i}$ has a half-normal distribution, $u_{i} \sim N^{+}\left(0, \sigma_{u}^{2}\right)$. With mean, $E(u)=\sigma_{u}(2 / \pi)^{1 / 2}$, variance, $\operatorname{Var}(u)=\sigma_{u}^{2}(\pi-2 / \pi)$ and composite error is $\varepsilon=v-u$, therefore the variance for $\varepsilon$ as below.

$$
\operatorname{Var}(\varepsilon)=\operatorname{Var}(u)+\operatorname{Var}(v)-2 \operatorname{Cov}(u, v)
$$

This mean that a positive correlation between $u$ and $v$ will reduces the variances of $\varepsilon$ and a negative correlation between $u$ and $v$ will increase the variance of $\varepsilon$ [11]. The joint density of $u$ and $v$ when they are assume as dependent can expressed for all $u_{i} \geq 0$ as:

$$
h(u, v)=g_{1}(u) g_{2}(v) c_{\theta}\left(G_{1}(u), G_{2}(v)\right)
$$

The joint density of $u$ and $\varepsilon$ is obtained by replacing $v$ in $h(u, v)$ by $v=\varepsilon+u$. To obtain the density of $\varepsilon$, the joint density of $u$ and $\varepsilon$ is then integrated by the variable $u$ as following:

$$
\begin{aligned}
h(\varepsilon) & =\int_{0}^{+\infty} h(u, \varepsilon) d u \\
& =\int_{0}^{+\infty} g_{1}(u) g_{2}(\varepsilon+u) c_{\theta}\left(G_{1}(u), G_{2}(\varepsilon+u)\right) d u \\
& =E_{U} g_{2}(\varepsilon+u) c_{\theta}\left(G_{1}(u), G_{2}(\varepsilon+u)\right)
\end{aligned}
$$

Replacing $\varepsilon=y-f(x, \beta)$ in the expression $h(\varepsilon)$ gives the density of $y$. Based on maximum likelihood estimator (MLE), one can address the issue of estimating technical efficiencies, defined as the expectation of conditional on observed residuals. Hence, technical efficiencies of DMUs which depend on the parameter $\theta=\left(\sigma_{u}, \sigma_{v}, \theta, \beta\right)$. Through observed input, $x$ and output $y$, the technical efficiencies, $T E$ is defined by:

$$
T E=E(\exp \{-u\} \mid \varepsilon)
$$

Using the marginal distribution of $\varepsilon$ in (5) and (6), and the joint density of $u$ and $\varepsilon, T E$ can calculate based on:

$$
T E=\int_{\Re^{+}} \exp \{-u\} h(u \mid \varepsilon) d u
$$




$$
=\frac{1}{h(\varepsilon)} \int_{\Re^{+}} \exp \{-u\} h(u \mid \varepsilon) d u
$$

If we assume the MLE of parameter $\theta=\left(\sigma_{u}, \sigma_{v}, \theta, \beta\right)$ obtain from (6) are $\theta_{M L}=\left(\hat{\sigma}_{u}, \hat{\sigma}_{v}, \hat{\theta}, \hat{\beta}\right)$, then the replacing these estimates in (7), we can obtain $T E_{M L}$ which is the MLE of $T E$.

\section{METHODOLOGY}

The study estimates the technical efficiencies for 12 construction companies listed at Bursa Malaysia. The sample dataset which includes asset turnover (AT), debt to equity ratio (D/E) and return on equity (ROE) for 12 companies was collected from Eikon Thomson Reuter's database. The selected construction listed companies are Ahmad Zaki Resources Bhd $\left(\mathrm{DMU}_{1}\right)$, Benalec Holdings Bhd $\left(\mathrm{DMU}_{2}\right)$, Bina Puri Holdings Bhd $\left(\mathrm{DMU}_{3}\right)$, Brem Holdings Bhd Industries Bhd $\left(\mathrm{DMU}_{6}\right)$, Ekovest Bhd $\left(\mathrm{DMU}_{7}\right)$, Eversendai Corporation Bhd $\left(\mathrm{DMU}_{8}\right)$, Fajarbaru Builder Group Bhd $\left(\mathrm{DMU}_{9}\right)$, Gadang Holdings Bhd $\left(\mathrm{DMU}_{10}\right)$, Mitrajaya Holdings Bhd $\left(\mathrm{DMU}_{11}\right)$ and Malaysian Resources Corporation Bhd (DMU $\left.\mathrm{DM}_{12}\right)$.

The fundamental concept of all frontier estimation methods is the comparison between the DMUs to understand how inputs are generating outputs and the comparison is based on each unit's TE score. Definition technical efficiency score represents the company's capacity to achieve maximum production output from the set of inputs. There are 2 inputs and 1 output for compute the efficiency score as follows:

\section{Input 1:}

AT was measured by the value of a company's sales and revenue in relation to the value of its assets and used as an indicator of the efficiency in which the assets were used to generate the revenue:

\section{Input 2:}

$\mathrm{D} / \mathrm{E}$ ratio is used in this study to evaluate a company's leverage;

\section{Output:}

The selection of ROE as output for this study is mainly based on the connection between efficiency and profitability that is known as DuPont Model [12].

DuPont Model is a useful tool for analyzing financial statement which the performance helps to predict future profitability.

Referring to (3), we consider the Cobb-Douglas production model as:

$$
\begin{aligned}
& \ln (\text { Return on Equity })_{i t}=\beta_{0}+\beta_{1} \ln (\text { Asset turnover })_{i t} \\
& \quad+\beta_{2} \ln (\text { Debt to equity })_{i t}+v_{i t}-u_{i t}
\end{aligned}
$$

The maximum likelihood is used to assess SFA model parameters as shown in (9) and SFA-copula model, where the copula functions are shown in the appendix section. We can estimate the parameters of the models and technical $\left(\mathrm{DMU}_{4}\right)$, Crest Builder Holding Bhd $\left(\mathrm{DMU}_{5}\right)$, DKLS

efficiency (TE) using the MATLAB software. However, in R-programming, the estimation of technical efficiency based on DEA models was calculated in combination with CCR and BCC models.

The parameter estimates and TEs are displayed in the next section. A technical efficiency value can either be 1 or less than 1 .

- The producers or DMUs at the frontier with technical efficiency of 1 can be called fully efficient.

- When they fail to reach the frontier, they are called production inefficiency and the technical efficiency score will be less than one.

Table 1: Selected copula families

\begin{tabular}{|c|c|c|c|}
\hline Family & $\begin{array}{c}\text { Generator, } \\
\ell_{\theta}(t)\end{array}$ & $\begin{array}{c}\text { Kendall's Tau } \\
\tau\end{array}$ & $\begin{array}{c}\text { Interval } \\
\text { for } \theta\end{array}$ \\
\hline Clayton & $\frac{1}{\theta}\left(\frac{1}{t^{\theta}}-1\right)$ & $\frac{\theta}{\theta+2}$ & $(0, \infty)$ \\
\hline Gumbel & $(-\ln t)^{\theta}$ & $\frac{\theta-1}{\theta}$ & {$[1, \infty)$} \\
\hline A12 & $\left(\frac{1}{t}-1\right)^{\theta}$ & $1-\frac{2}{3 \theta}$ & {$[1, \infty)$} \\
\hline
\end{tabular}

\section{RESULTS AND DISCUSSION}

This research was carried out on 12 construction companies in Malaysia. The maximum likelihood method was used in Cobb-Douglas function to calculate the parameters. The estimated parameters for SFA and SFA-copula models are given in Table 2.

Table 2: The estimated parameters for SFA model

\begin{tabular}{|c|c|c|c|c|c|c|}
\hline $\mathbf{F}$ & $\sigma_{u}$ & $\sigma_{v}$ & $\theta$ & $\beta_{0}$ & $\beta_{1}$ & $\beta_{2}$ \\
\hline \multirow{2}{*}{$\mathrm{C}$} & 0.320 & 0.164 & 5.563 & 5.486 & -0.186 & 0.830 \\
& 4 & 4 & 7 & 5 & 3 \\
\hline \multirow{2}{*}{$\mathrm{G}$} & 0.078 & 0.355 & 1.731 & 9.291 & -0.415 & 0.701 \\
& 2 & 9 & 9 & 7 & 0 & 8 \\
\hline \multirow{2}{*}{$\mathrm{A}$} & 0.142 & 0.112 & 7.274 & 4.019 & -0.098 & 0.879 \\
& 7 & 4 & 1 & 2 & 5 & 6 \\
\hline \multirow{2}{*}{$\mathrm{P}$} & 11.50 & 0.105 & - & 3.847 & -0.087 & 0.884 \\
& 7 & 3 & & 1 & 1 & 7 \\
\hline \multirow{2}{*}{$\mathrm{S}$} & 0.915 & 0.579 & - & 13.74 & -0.684 & 0.554 \\
& 3 & 7 & - & 3 & 8 & 6 \\
\hline
\end{tabular}

Notations: $\mathbf{F}=$ Family $; \mathrm{C}=$ Clayton; $\mathbf{G}=$ Gumbel; $\mathrm{A}=$
A12;
$\mathbf{P}=$ Product; $\mathbf{S}=$ Standard SFA

Table 2 and 3 show the dependence parameters, $\theta$ and Kendall's $\tau$. Kendall's $\tau$ is the probability of concordance minus the probability of discordance, and is thus standardized to the interval $[-1,1]$.

Table 3: Parameter Kendall's, $\tau$ for each family

\begin{tabular}{|c|c|c|c|c|c|}
\hline $\mathbf{F}$ & $\mathbf{C}$ & $\mathbf{G}$ & $\mathbf{A}$ & $\mathbf{P}$ & $\mathbf{S}$ \\
\hline$\tau$ & 0.7356 & 0.4260 & 0.9084 & - & - \\
\hline
\end{tabular}

Notations: $\mathbf{F}=$ Family $; \mathrm{C}=$ Clayton; $\mathbf{G}=$ Gumbel; $\mathbf{A}=$ A12; $\mathbf{P}=$ Product; $\mathbf{S}=$ Standard SFA 
Kendall's $\tau$ has a direct relationship with copula interpret the degree of association between technical inefficiency error, $u$ and random noise error, $v$ based on Kendall's $\tau$ parameter value.

For the Clayton copula, Kendall's $\tau$ is given by $\tau=\frac{\theta}{\theta+1}$. $\tau=0.7356$ indicate that that there is a strong relationship between $u$ and $v$. Similarly, for Gumbel copula, the parameter $\theta=1.7319$ with Kendall's $\tau$ value of 0.4260 indicates moderate relationship between $u$ and $v$. The strongest association is shown by A12 family with $\tau=0.9084$. As discussed above, a positive correlation between $u$ and $v$ decreases the composite error variance, while a negative correlation between $u$ and $v$ raises the variance of $\varepsilon$. It means that in the mentioned model in (9), the minimum variance tends the model to the closest estimation. Furthermore, from Table 2, the ratio of standard deviations lambda, $\lambda$ can estimated by $\lambda=\sigma_{u} / \sigma_{v}$. It measures proportion of composite error distribution. For instance, the Clayton copula will have $\lambda$ of 1.9537 . The coefficient of the parameter gamma as in shown (10) provides information regarding the presence of efficiency in the production.

$$
\gamma=\frac{\sigma_{u}^{2}}{\sigma_{u}^{2}+\sigma_{v}^{2}}=\frac{\lambda^{2}}{\lambda^{2}+1}
$$

The parameter $\gamma$ lies between 0 and 1 where $\gamma=0$ specifies that all frontier deviations are due to random error (noise), while $\gamma=1$ means all deviations are resulted from the technical inefficiency. For example, for standard SFA, value of gamma is 0.7137 . Since this value is close to one, the variation comes mainly from technical inefficiency. Tables 4 , 5 and 6 provide the efficiency score (ES) for each construction company or DMU. The ranking of DMU based on ES is also shown in the tables.

Specifically, Table 4 provides the ES and rank of DMU for SFA-copula models (Clayton, A12 and Gumbel). The maximum efficiency score for Clayton copula is 0.9973 and minimum score are 0.0948. For Gumbel copula and A12 copula, the maximum efficiency score is 1 and minimum value are 0.0964 and 0.0917 respectively. The rankings of DMUs across the SFA-copulas do not show significant difference.

Table 5 reports the ES and rank for Product copula and standard SFA model. Comparing for both groups, standard SFA has the lowest ES of 0.0870. Referring to Table 4 and Table 5, the top rank is $\mathrm{DMU}_{5}$ for Clayton, Gumbel and A12. However, for bottom rank is $\mathrm{DMU}_{12}$ for Clayton, Gumbel, A12, Product and Standard SFA. All results from Table 4 and Table 5 are compared with efficiency performance provided in Table 6, where ES are measured by DEA-CCR and DEA-BCC models. Table 6 provides efficiency scores produced by non-parametric frontier technique, DEA model with CCR and BCC models. The CCR model evaluates both technical and scale efficiency and therefore the general technical efficiency can be calculated by combining both measures in a single efficiency score. However, the pure parameter, $\theta$ as shown in Table 1. Therefore, we can Hence, the estimated $\theta=5.5634$ has the value of

technical efficiency of DMUs is evaluated for the BCC model which is called local technical efficiency.

Based on Table 7, Gumbel and Clayton has the highest correlation value (0.9930) which indicates strong relationship between the models. Clayton, Gumbel and A12 copulas have high correlations with each other. This results may be due to the fact that they belong to Archimedean copula family. The minimum coefficient value is between Standard SFA model and DEA-BCC model with 0.0839. According to [12], the best parametric model can be determined if it has the highest correlation with the DEA-CCR model. The model that has the highest correlation with CCR model is Product copula with a coefficient value of 0.9371 . The standard SFA model has the lowest correlation with the CCR model where the coefficient value is 0.5874 .

Therefore, based on Table 7, Product copula can be said as a relatively good model for the dataset since it has the highest correlation with the CCR model. The alternative approach for determining the best model to estimate efficiency scores is by checking the information criterion (AIC or BIC) [13], [14]. This approach will be conducted in the next study.

Table 4: Efficiency score for Clayton, A12 and Gumbel

\begin{tabular}{|c|c|c|c|c|c|c|}
\hline \multirow{2}{*}{$\begin{array}{l}\mathbf{D} \\
\mathbf{M} \\
\mathbf{U}\end{array}$} & \multicolumn{2}{|c|}{ Clayton } & \multicolumn{2}{|c|}{ Gumbel } & \multicolumn{2}{|l|}{ A12 } \\
\hline & ES & Rank & ES & Rank & ES & Rank \\
\hline 1 & 0.1763 & 10 & 0.1590 & 10 & 0.2330 & 10 \\
\hline 2 & 0.8678 & 4 & 0.9893 & 3 & 0.6227 & 5 \\
\hline 3 & 0.1357 & 11 & 0.1148 & 11 & 0.2115 & 11 \\
\hline 4 & 0.4346 & 7 & 0.4845 & 7 & 0.3307 & 8 \\
\hline 5 & 0.9973 & 1 & 1 & 1 & 1 & 1 \\
\hline 6 & 0.8868 & 3 & 0.8869 & 4 & 0.8945 & 4 \\
\hline 7 & 0.6156 & 6 & 0.6714 & 6 & 0.4960 & 6 \\
\hline 8 & 0.4272 & 8 & 0.4093 & 8 & 0.4820 & 7 \\
\hline 9 & 0.2766 & 9 & 0.2958 & 9 & 0.2344 & 9 \\
\hline 10 & 0.8324 & 5 & 0.8079 & 5 & 0.9075 & 3 \\
\hline 11 & 0.9971 & 2 & 0.9993 & 2 & 1 & 2 \\
\hline 12 & 0.0948 & 12 & 0.0964 & 12 & 0.0917 & 12 \\
\hline
\end{tabular}

Notation: $E S=$ Efficiency score

Table 5: Efficiency score for product copula and standard SFA model

\begin{tabular}{|l|l|l|l|l|}
\hline \multirow{2}{*}{ DMU } & Product & \multicolumn{2}{l|}{ Standard SFA } \\
\cline { 2 - 5 } & ES & Rank & ES & Rank \\
\hline 1 & 0.1565 & 10 & 0.3200 & 1 \\
\hline 2 & 1 & 1 & 0.4219 & 6 \\
\hline 3 & 0.1122 & 11 & 0.3517 & 8 \\
\hline 4 & 0.4892 & 7 & 0.2385 & 10 \\
\hline 5 & 0.9981 & 2 & 0.9918 & 3 \\
\hline 6 & 0.8837 & 4 & 0.8981 & 4 \\
\hline 7 & 0.6771 & 6 & 0.3807 & 7 \\
\hline 8 & 0.4057 & 8 & 0.5514 & 5 \\
\hline 9 & 0.2963 & 9 & 0.1936 & 11 \\
\hline 10 & 0.8016 & 5 & 1 & 1 \\
\hline 11 & 0.9952 & 3 & 1 & 2 \\
\hline 12 & 0.0965 & 12 & 0.0870 & 12 \\
\hline
\end{tabular}


Table 6: Efficiency score for DEA models

\begin{tabular}{|l|l|l|l|l|}
\hline \multirow{2}{*}{ DMU } & \multicolumn{2}{|l|}{ DEA-CCR } & \multicolumn{2}{l|}{ DEA-BCC } \\
\cline { 2 - 5 } & ES & Rank & ES & Rank \\
\hline 1 & 0.1365 & 11 & 0.2215 & 11 \\
\hline 2 & 1 & 1 & 1 & 1 \\
\hline 3 & 0.0882 & 12 & 0.1571 & 12 \\
\hline 4 & 0.5830 & 7 & 1 & 2 \\
\hline 5 & 1 & 2 & 1 & 3 \\
\hline 6 & 0.8888 & 5 & 0.9043 & 7 \\
\hline 7 & 1 & 3 & 1 & 4 \\
\hline 8 & 0.3837 & 8 & 0.4324 & 10 \\
\hline 9 & 0.5857 & 9 & 1 & 5 \\
\hline 10 & 0.7279 & 6 & 0.7726 & 8 \\
\hline 11 & 0.9295 & 4 & 1 & 6 \\
\hline 12 & 0.2196 & 10 & 0.7333 & 9 \\
\hline
\end{tabular}

Table 7: Correlation between selected models

\begin{tabular}{|l|l|l|l|l|l|l|l|}
\hline & $\begin{array}{l}\text { Clay } \\
\text { ton }\end{array}$ & $\begin{array}{l}\text { Gum } \\
\text { bel }\end{array}$ & A12 & $\begin{array}{l}\text { Prod } \\
\text { uct }\end{array}$ & $\begin{array}{l}\text { Std. } \\
\text { SF } \\
\text { A }\end{array}$ & $\begin{array}{l}\text { CC } \\
\text { R }\end{array}$ & $\begin{array}{l}\text { BC } \\
\text { C }\end{array}$ \\
\hline $\begin{array}{l}\text { Clayt } \\
\text { on }\end{array}$ & 1.000 & 0.993 & 0.97 & 0.958 & 0.79 & 0.88 & 0.60 \\
\hline Gum & 0.993 & 1.000 & 0.96 & 0.979 & 0.78 & 0.90 & 0.64 \\
bel & 0 & 0 & 50 & 0 & 32 & 91 & 33 \\
\hline A12 & 0.972 & 0.965 & 1.00 & 0.916 & 0.88 & 0.83 & 0.48 \\
& 0 & 0 & 00 & 1 & 81 & 22 & 95 \\
\hline Prod & 0.958 & 0.979 & 0.91 & 1.000 & 0.73 & 0.93 & 0.69 \\
uct & 0 & 0 & 61 & 0 & 43 & 71 & 23 \\
\hline Std. & 0.797 & 0.783 & 0.88 & 0.734 & 1.00 & 0.58 & 0.08 \\
SFA & 2 & 2 & 81 & 3 & 00 & 74 & 39 \\
\hline CCR & 0.881 & 0.909 & 0.83 & 0.937 & 0.58 & 1.00 & 0.79 \\
& 1 & 1 & 22 & 1 & 74 & 00 & 02 \\
\hline BCC & 0.601 & 0.643 & 0.48 & 0.692 & 0.08 & 0.79 & 1.00 \\
& 4 & 3 & 95 & 3 & 39 & 02 & 00 \\
\hline
\end{tabular}

\section{CONCLUSION}

The stochastic frontier analysis (SFA) model is a linear regression model, in general, and has two component errors, $u$ and $v$, where the errors are often assumed to be independent. This study uses the Cobb-Douglas SFA model (standard SFA) to estimate the technical efficiencies (TEs) of 12 publicly listed construction companies. Due to the error independence assumption in the standard SFA model, this study also estimates TEs based on the SFA-copula models where the copula component relaxes the dependency assumption between the two error components. In addition, TEs were also produced from Data Envelopment Analysis (DEA) models, the non-parametric frontier technique, namely DEA-CCR and DEA-BCC. The comparison of TEs for all models used in this study were displayed together with the ranks of the companies (DMUs).

This study reveals slight differences in TEs produced by SFA-copula models. However, in terms of the ranking, the positions of DMUs showed are almost the same for each model. In fact, SFA-Product Copula model can be named as a best model in this context since it has the highest correlation with the DEA-CCR model. Meanwhile, the standard SFA model showed obvious differences in TEs and DMU rankings compared to SFA-copula, DEA-CCR and DEA-BCC model. This study also found that the standard SFA model is not a good model due to the lowest correlation with DEA-CCR model. In conclusion, the neglecting of the error dependence assumption in an SFA model will result false estimations of parameters and efficiency scores.

Future study can further support the findings of the present study by using information criterion as the basis to determine the best SFA models for the dataset.

\section{APPENDIX}

The definition of copula and properties of selected copulas are extracted from [15], [16].

\section{Definition Copula}

Copula can be defined informally as follows:

Let $U$ and $V$ be continuous random variables with distribution function:

$G_{1}(u)=P(U \leq u)$ and $G_{2}(v)=P(V \leq v)$

The joint distribution function is:

$h(u, v)=P(U \leq u, V \leq v)$.

For every $(u, v)$ in $[-\infty, \infty]^{2}$ consider the point in $I^{3}$ $(I=[0,1])$ with coordinates $\left(G_{1}(u), G_{2}(v), h(u, v)\right)$.

This mapping from $I^{2}$ to $I$ is a copula. Copula are also known as dependence functions or uniform representations.

A two-dimensional copula is a function $c=I^{2} \rightarrow I$ such that:

C1:

for every $u, v$ in $I$,

$c(u, 0)=0=c(0, v) ; c(u, 1)=u$ and $c(1, v)=v$;

C2:

For every $u_{1}, u_{2}, v_{1}, v_{2}$ in $I$ such that $u_{1} \leq u_{2}$ and $v_{1} \leq v_{2}$.

\section{Product copula}

The function $\Pi(u, v)=u . v$ is called the product copula and has an important statistical interpretation.

Let $u=G_{1}(u)$ and $v=G_{2}(v)$

The product copula is important as benchmark because it correspond to independence.

It is easy to see that function $\Pi(u, v)=u . v$ satisfies conditions (C1) and (C2), and hence is a copula.

\section{Clayton copula}

Copula of the form $c(u, v)=\ell^{-1}(\ell(u)+\ell(v))$ is said to be Archimedean and function $\ell$ is said to be its generator.

The Clayton family can reflect lower tail dependence for $\theta>0$.It is characterized by the following formula:

$c_{\theta}(u, v)=\left[\max \left(u^{-\theta}+v^{-\theta}-1,0\right)\right]^{-1 / \theta}$

This copula can only capture a strong lower tail and positive dependence, but it can be rotated and used in capture negative dependence or reflect strong upper tail dependence.

The lower tail dependence coefficient is $\lambda_{L}=2^{-1 / \theta}$.

Published By:

Blue Eyes Intelligence Engineering 


\section{Gumbel copula}

The bivariate Gumbel copula is given by:

$$
c_{\theta}(u . v)=\exp \left(-\left[(-\ln u)^{\theta}+(-\ln v)^{\theta}\right]^{1 / \theta}\right)
$$

It is an asymmetric Archimedean copula that allows for strong upper tail dependence.

The upper tail dependence coefficient is $\lambda_{U}=2-2^{1 / \theta}$.

\section{A12}

The bivariate $\mathrm{A} 12$ copula is given by:

$$
c_{\theta}(u, v)=\left(1+\left[\left(u^{-1}-1\right)^{\theta}+\left(v^{-1}-1\right)^{\theta}\right]^{1 / \theta}\right)^{-1}
$$

The upper tail dependence coefficient $\lambda_{U}=2-2^{1 / \theta}$ and lower tail dependence coefficient is $\lambda_{L}=2-2^{-1 / \theta}$.

\section{ACKNOWLEDGMENT}

The authors are indebted to reviewers for their valuable remarks and suggestions, leading to the improvement of the paper. Also thanks to Faculty of Computer and Mathematical Sciences (FSKM), Universiti Teknologi MARA, Perak Branch on the financial research funding.

\section{REFERENCES}

1. A. Carta and M. F. Steel, "Modelling multi-output stochastic frontiers using copulas," Computational Statistics and Data Analysis, 56(11), 2012, pp. 3757-3773.

2. L. R. Murillo-Zamorano, "Economic efficiency and frontier techniques," Journal of Economic Surveys, 18(1), 2004, pp. 33-77.

3. D. Aigner, C. K. Lovell, and P. Schmidt, "Formulation and estimation of stochastic frontier production function models," Journal of Econometrics, 6(1), 1977, pp. 21-37.

4. W. Meeusen and J. van Den Broeck, "Efficiency estimation from Cobb-Douglas production functions with composed error," International Economic Review, 18(2), 1977, pp. 435-444.

5. P. Schmidt and C. K. Lovell, "Estimating stochastic production and cost frontiers when technical and allocative inefficiency are correlated," Journal of Econometrics, 13(1), 1980, pp. 83-100.

6. M. Pal and A. Sengupta, " A model of FPF with correlated error components: An application to Indian agriculture," Indian Journal of Statistics, 61(B), 1999, pp. 337-350.

7. A. Das, "Copula-based stochastic frontier model with autocorrelated inefficiency," Central European Journal of Economic Modelling and Econometrics, 7, 2015, pp. 111-126.

8. M. D. Smith, "Stochastic frontier models with dependent error components," Econometrics Journal, 11(1), 2008, pp. 172-192.

9. A. Wiboonpongse, J. Liu, S. Sriboonchitta, and T. Denoeux, "Modeling dependence between error components of the stochastic frontier model using copula: application to intercrop coffee production in Northern Thailand," International Journal of Approximate Reasoning, 65, 2015, pp. 34-44.

10. G. E. Battese and T. J. Coelli, "Frontier production functions, technical efficiency and panel data: with application to paddy farmers in India," Journal of Productivity Analysis, 3(1-2), 1992, pp. 153-169.

11. R. E. Mehdi and C. M. Hafner, "Inference in stochastic frontier analysis with dependent error terms," Mathematics and Computers in Simulation, 102 2014, pp. 104-116.

12. S. C. Sheela and K. Karthikeyan, "Financial performance of pharmaceutical industry in India using DuPont analysis," European Journal of Business and Management, 4(14), 2012, pp. 84-91.

13. S. Sriboonchitta, J. Liu, A. Wiboonpongse, and T Denoeux, "A double-copula stochastic frontier model with dependent error components and correction for sample selection," International Journal of Approximate Reasoning, 80, 2017, pp. 174-184.

14. P. Tibprasorn, S. Chanaim, and S. Sriboonchitta, " A copula-based stochastic frontier model and efficiency analysis: Evidence from stock exchange of Thailand," International Symposium on Integrated Uncertainty in Knowledge Modelling and Decision Making, 2016, pp. 637-648.

15. R. B. Nelsen, An Introduction to Copulas. Berlin: Springer Science and Business Media, 2007.

16. R. B. Nelsen, Dependence modeling with Archimedean copulas. 2005, Available: http://web.pdx.edu/ fountair/seminar/arch.pdf. 